\title{
Effectiveness of Telehealth Acceptance and Commitment Therapy for Generalized Anxiety Disorder During the COVID-19 Pandemic: Protocol for a Systematic Review and Meta-Analysis
}

\section{Ruiying Jia}

Henan University https://orcid.org/0000-0001-9869-559X

Jingfen Yu

Henan University

Dandan Liang

Henan University

Zhen Wu

Henan University

Chaoran Chen ( $\nabla$ kfccr@126.com )

Henan University https://orcid.org/0000-0002-6237-2999

Guangli Lu

Henan University

\section{Protocol}

Keywords: telehealth, acceptance and commitment therapy, generalized anxiety disorder, protocol, systematic review, meta-analysis, coronavirus disease 2019

Posted Date: July 2nd, 2021

DOI: https://doi.org/10.21203/rs.3.rs-666275/v1

License: (c) (i) This work is licensed under a Creative Commons Attribution 4.0 International License. Read Full License 


\section{Abstract}

Background: Generalized anxiety disorder (GAD) is a highly prevalent, chronic, costly and disabling mental disorder. However, due to various reasons, patients are not treated effectively. During the COVID19 pandemic, when face-to-face intervention is limited to a certain extent, researchers have highlighted the need for evidence-based interventions that can be delivered remotely, such as telehealth mediated acceptance and commitment therapy (ACT).

Methods/design: We will systematically search PubMed, Embase, Cochrane Library, EBSCO (MEDLINE, Psychology \& Behavioral Sciences Collection), Web of Science, CNKI, and WanFang Database for randomized controlled trials examining telehealth acceptance and commitment therapy for generalized anxiety disorder. The Cochrane Collaboration's bias assessment tool will be used to evaluate the risk of bias for the included studies, RevMan 5.3 and Stata 14.0 will be utilized for the meta-analysis.

Discussion: This study will provide evidence for evaluating the efficacy of telehealth acceptance and commitment therapy for generalized anxiety disorder treatment during the COVID-19 pandemic. All of these results may be used to improve clinical decision-making and future studies.

\section{Systematic review registration: PROSPERO CRD42021255909.}

\section{Background}

Generalized anxiety disorder (GAD) constitutes one of the most common groups of mental illnesses in adults, characterized by excessive anxiety and worry, as well as associated symptoms such as restlessness, fatigue, irritability, muscle tension, and/or sleep disturbance [1]. GAD is a chronic disorder with episode duration commonly averaging a decade or longer [2]. What's more, there is high comorbidity of GAD with other anxiety and mood disorders [3]. The global lifetime prevalence of GAD was estimated at $3.7 \%$ and 12 -month prevalence at $1.8 \%$ [4]. Studies showed that GAD is associated with poor quality of life [5], increased health care utilization [6], impaired memory [7], high risk for death by suicide [8]. As is known to all, Coronavirus disease (COVID-19), declared a pandemic on 11 March 2020 by the World Health Organization, changed the lifestyle of a large number of people and placed a significant strain on health systems on a large scale. Studies suggested that people with a previous history of anxiety disorder or other mental disorders are at high risk of symptom worsening during the COVID-19 pandemic because they are more vulnerable than the general population to the fear of getting sick and to the changes in lifestyle related to quarantine $[9,10]$.Therefore, it is urgent to find effective treatment measures for a series of harms caused by GAD.

Medications and psychotherapy are the primary treatments for GAD [11]. Although pharmacological treatments have demonstrated some success in treating GAD, they are associated with some potentially serious adverse effects [12]. Common adverse reactions include increased nervousness, insomnia, nausea and sexual dysfunction, among others [13]. Furthermore, many patients fail to respond to or cannot tolerate currently recommended pharmacotherapies [14]. It was reported that many patients have 
a preference for psychological treatments over pharmacological approaches [15]. Some psychological therapies have proven efficacy in the treatment of generalised anxiety disorder and the most common used is cognitive behavioural therapy (CBT) [16]. Acceptance and Commitment Therapy (ACT), whose ultimate goal is to improve psychological flexibility, is a representative treatment in the third wave of CBT [17].

ACT is a psychological therapy that encourages participants to change their relationships with their thoughts and physical sensations through mechanisms of acceptance, mindfulness and value-based action [18]. The treatment focuses on acceptance as a process resulting in increased psychological flexibility that works as a buffer against experiential avoidance and ineffective coping [19]. The purpose of ACT is not to challenge distressing thoughts or feelings, as it is in CBT, but rather to allow them to be present, with an understanding that distress is a natural part of life that no need to fight against and increase engagement in meaningful life activities [20].

There was preliminary evidence of good feasibility and acceptability of ACT for people with GAD [21, 22]. Unfortunately, such interventions may be inaccessible for many people, due to difficulties obtaining and attending appointments from remote locations, the high cost of treatment, long waiting lists, lack of clinician training, time restraints, or unavailability through health services [23]. To make psychological treatment accessible to the broader population, researchers have developed telehealth interventions [24]. Since the COVID-19 pandemic began, the reach of telehealth services, including internet and telephone coaching, has expanded exponentially [25]. Telehealth services have overcome physical barriers and maintained continuity of medical care for patients during the pandemic $[26,27]$.

Evidence indicates that telehealth ACT can be a feasible and potentially efficacious treatment for GAD [28]. However, there is currently no meta-analysis on telehealth ACT intervention for patients with GAD. Therefore, this systematic review and meta-analysis will aim at evaluating the efficacy of telthealth ACT in the treatment of GAD and to provide evidence-based information for clinical applications during the COVID-19 pandemic.

\section{Methods/design}

\section{Study registration}

This systematic review protocol has been registered in PROSPERO, and the registration number is CRD42021255909. We strictly abide by the Preferred Reporting Items for Systematic Review and MetaAnalysis Protocols (PRISMA-P) guidelines [29]. PRISMA-P checklist has been provided in Additional file 1.

\section{Inclusion criteria}

Population: Population with a confirmed clinical diagnosis of GAD.

Interventions: In the intervention group, participants received telehealth ACT based on mobile phone, Internet or videoconferencing. 
Comparators: The control group included blank, placebo, usual or standard care, waiting list control, or drug therapy.

Outcomes: The studies assessed measurable anxiety.

Studie design: This study will include randomized controlled trials (RCTs) investigating the effect of telehealth ACT on generalized anxiety disorder during the COVID-19 pandemic.

Language: In this systematic review, we only include articles published in English and Chinese.

\section{Search strategy}

The following electronic databases were utilized for selecting eligible studies published from inception to May 2021: PubMed, Embase, Cochrane Library, EBSCO (MEDLINE, Psychology \& Behavioral Sciences Collection), Web of Science, CNKI, and WanFang Database. The search strategy in PubMed is as follows:

\#1 Search: "Acceptance and Commitment Therapy"[Mesh] Sort by: Most Recent

\#2 Search: "Telemedicine"[Mesh] Sort by: Most Recent

\#3 Search: $((((((($ Mobile Health[Title/Abstract]) OR (mHealth[Title/Abstract])) OR

(Telehealth[Title/Abstract])) OR (eHealth[Title/Abstract])) OR (remote[Title/Abstract])) OR (internet[Title/Abstract])) OR (telephone[Title/Abstract])) OR (smartphone[Title/Abstract])) OR (video conferenc[Title/Abstract]) Sort by: Most Recent

\#4 \#2 OR \#3

\#5 Search: generalized anxiety disorder[Title/Abstract] Sort by: Most Recent

\#6 Search: "COVID-19"[Mesh] Sort by: Most Recent

\#7 Search: $((((((((((C O V I D) 19[$ Title/Abstract]) OR (COVID-19 Virus Disease[Title/Abstract])) OR (COVID-19 Virus Infection[Title/Abstract])) OR (2019-nCoV Infection[Title/Abstract])) OR (Coronavirus Disease19[Title/Abstract])) OR (2019 Novel Coronavirus Disease[Title/Abstract])) OR (2019 Novel Coronavirus Infection[Title/Abstract])) OR (2019-nCoV Disease[Title/Abstract])) OR (Coronavirus Disease 2019[Title/Abstract])) OR (SARS Coronavirus 2 Infection[Title/Abstract])) OR (SARS-CoV-2 Infection[Title/Abstract])) OR (COVID-19 Pandemic[Title/Abstract]) Sort by: Publication Date

\#8 Search: \#6 OR \#7

\#9 Search: ((randomized controlled trial[Title/Abstract]) OR (controlled clinical trials, randomized[Title/Abstract])) OR (RCT[Title/Abstract]) Sort by: Publication Date \#10 Search: \#1 AND \#4 AND \#5 AND \#8 AND \#9 


\section{Study selection process}

Two independent reviewers will screen and evaluate the relevant abstracts and titles of all studies based on pre-defined inclusion criteria and then exclude ineligible articles with the reasons. The third investigator will solve any disagreement between the two reviewers. The process of screening selection is shown in Figure 1.

We will utilize EndNote X9 software to reject duplicates. Following this, the two reviewers will screen the titles and abstracts of the included trials. If there are insufficient information to make a decision based on the title and abstract, the full article will be obtained for review. The full text of articles will be reviewed by the two reviewers based on the inclusion criteria. If the eligibility cannot be determined using the reported information, we will contact authors for clarification.

\section{Data extraction and analysis}

Two authors will independently collect data from all studies based on the data extraction form. The following study information will be recorded: first author, year of publication, country, sample size, participant age, recruitment site, intervention duration and mode, outcome measures, specific treatment for the control groups, follow-up time and intervention content. The third author will resolve any disagreement between the two authors.

\section{Dealing with missing data}

The accuracy of the data may affect the results of the study, and even lead to different conclusions. Therefore, we will contact the corresponding authors to supplement the research results with insufficient or missing data. If the corresponding author cannot be contacted, the data will be processed separately, and the potential impacts of incomplete data on the results will be analyzed and reported as well.

\section{Risk of bias assessment}

We will use the tool recommended by the Cochrane Handbook Version 5.1.0 [30] to analyze the risk of bias in the trials from the following seven aspects: random sequence generation, allocation concealment, blinding of participants and personnel, blinding of outcome assessment, incomplete outcome data, selective reporting, and other bias. Every item will be classified as yes (low risk of bias), no (high risk of bias), or unclear (moderate risk of bias). When the risk of bias of all seven components are defined as low risk of bias, the trial will be defined as the overall low risk of bias. At the same time, when one or more of the seven bias components are classified as high risk, the trial will be graded as high risk of bias. In other cases, the trial will be graded as unclear risk. Disagreements in bias classification will be resolved by discussions among the two reviewers and, if necessary, through discussions with the authors.

\section{Measures of treatment effects.}

Standardized mean difference is applied to measure the efficacy of $95 \%$ confidence interval. 


\section{Assessment of quality of evidence}

Two independent reviewers will assess the quality of evidence on outcome level using the Grading of Recommendations Assessment, Development and Evaluation (GRADE) [31]. The level of evidence will be divided into high, moderate, low, and very low quality. Potential discrepancies between the GRADE ratings will be resolved by a third researcher.

\section{Assessment of heterogeneity}

According to the Cochrane Handbook, Chi-Squared test and $R$ value could be used to evaluate the heterogeneity. $R$ values of $25 \%, 50 \%$, and $75 \%$ are considered as low, moderate, and high heterogeneity, respectively.

\section{Assessment of publication bias}

We will perform funnel plots and visually examine the signs of asymmetry to investigate publication bias, then use Egger's test [32] as a formal test of publication bias when the number of the included studies was more than $10(n \geq 10)$.

\section{Data synthesis}

We will enter group means, standard deviations (SD), and the number of participants in RevMan 5.3 and conduct a random-effects model meta-analysis, and Stata 14.0 will be used for sensitivity analysis and Egger's test.

\section{Subgroup analysis}

If available, we will conduct subgroup analyses based on different interventions modes, intervention time, age of participants, different countries, and outcome measures.

\section{Sensitivity analysis}

We will use sensitivity analysis to examine the stability of the results by removing individual trials to determine whether the removed study had a particular effect.

\section{Discussion}

Generalized anxiety disorder (GAD) is a highly prevalent, chronic, costly and disabling mental disorder[33]. However, due to various reasons (such as stigma, difficulty in accessing psychotherapy, long waiting times for appointments, living in remote areas, etc.), patients are not treated effectively[34, 35]. The COVID-19 explosion has made it more difficult to implement face-to-face psychological interventions. Studies have shown that telehealth technologies have great potential in providing health services to patients with $\operatorname{GAD}[36,37]$. The results of some clinical trials suggest that telehealth ACT may be effective in treating $\operatorname{GAD}[38,39]$. However, confidence in the effectiveness of telehealth ACT is limited because of 
the small effect size, the low level of treatment adherence, and the possibility of imprecise effect estimates. In addition, it remains unclear whether treatment effect can be predicted by characteristics of participants or interventions. Thus, the systematic review outlined in this protocol aims to identify, assess and synthesise using meta-analytic methods available in the evidence of the effects of telehealth ACT for the management of patients with GAD.

To the best of our knowledge, this is the first planned systematic review and meta-analysis that will review the field of telehealth ACT for patients with GAD during the COVID-19 pandemic. This systematic review will help tailor telehealth ACT interventions to more effectively meet the needs of GAD patients. This systematic review could also motivate other researchers to construct and test in randomised trials new or modified internet-based or mobile-based interventions for patients with GAD during the COVID-19 pandemic.

\section{Abbreviations}

ACT: Acceptance and Commitment therapy; GAD: generalized anxiety disorder; RCTs: randomized controlled trials; COVID-19: coronavirus disease 2019

\section{Declarations}

\section{Ethics approval and consent to participate}

Not applicable

Consent for publication

Not applicable

\section{Availability of data and materials}

Not applicable

\section{Competing interests}

The authors declare that they have no competing interests

\section{Funding}

The study was supported by Henan Province Graduate Education Reform and Quality Improvement Project (YJS2021AL074).

\section{Author Contributions:}


RYJ and JFY drafted the study protocol, conducted literature searching and wrote the manuscript. DDL and ZW conducted the literature searching and revised the manuscript. GLL and CRC supervised the manuscript writing process and revised the manuscript. All the authors approved the final version of the manuscript.

\section{Acknowledgements}

The study was supported by Henan Province Graduate Education Reform and Quality Improvement Project (YJS2021AL074). The authors thank all the other members of the team for their contributions to this study.

\section{References}

1. American Psychiatric Association. Diagnostic and statistical manual of mental disorders (5th ed.). 2013: Diagnostic and statistical manual of mental disorders (5th ed.).

2. Keller, MB. The long-term clinical course of generalized anxiety disorder. J Clin Psychiatry. 2002; 63 Suppl 8:11-6.

3. Price, M, AC Legrand, ZMF BrierL Hébert-Dufresne.The symptoms at the center: Examining the comorbidity of posttraumatic stress disorder, generalized anxiety disorder, and depression with network analysis. J Psychiatr Res. 2019; 109:52-58.

4. Ruscio, AM, LS Hallion, CCW Lim, S Aguilar-Gaxiola, A Al-Hamzawi, J Alonso, et al.Cross-sectional Comparison of the Epidemiology of DSM-5 Generalized Anxiety Disorder Across the Globe. JAMA Psychiatry. 2017; 74:465-475.

5. Revicki, DA, K Travers, KW Wyrwich, H Svedsäter, J Locklear, MS Mattera, et al.Humanistic and economic burden of generalized anxiety disorder in North America and Europe. J Affect Disord. 2012; 140:103-12.

6. Porensky, EK, MA Dew, JF Karp, E Skidmore, BL Rollman, MK Shear, et al.The burden of late-life generalized anxiety disorder: effects on disability, health-related quality of life, and healthcare utilization. Am J Geriatr Psychiatry. 2009; 17:473-82.

7. Mantella, RC, MA Butters, MA Dew, BH Mulsant, AE Begley, B Tracey, et al.Cognitive impairment in latelife generalized anxiety disorder. Am J Geriatr Psychiatry. 2007; 15:673-9.

8. Gilmour, H.Threshold and subthreshold Generalized Anxiety Disorder (GAD) and suicide ideation. Health Rep. 2016; 27:13-21.

9. Chatterjee, SS, CM BarikarA Mukherjee.Impact of COVID-19 pandemic on pre-existing mental health problems. Asian J Psychiatr. 2020; 51:102071. 
10. Yao, H, JH, Chen YF Xu.Patients with mental health disorders in the COVID-19 epidemic. Lancet Psychiatry. 2020; 7:e21.

11. Chen, TR, HC Huang, JH Hsu, WC OuyangKC Lin.Pharmacological and psychological interventions for generalized anxiety disorder in adults: A network meta-analysis. J Psychiatr Res. 2019; 118:73-83.

12. Reinhold, JA, LA Mandos, K RickelsFW Lohoff.Pharmacological treatment of generalized anxiety disorder. Expert Opin Pharmacother. 2011; 12:2457-67.

13. BaldwinS D.Evidence-based guidelines for the pharmacological treatment of anxiety disorders: recommendations from the British Association for Psychopharmacology. Journal of Psychopharmacology. 2005; 19:567-596.

14. Gomez, AF, AL BarthelSG Hofmann.Comparing the efficacy of benzodiazepines and serotonergic antidepressants for adults with generalized anxiety disorder: a meta-analytic review. Expert Opin Pharmacother. 2018; 19:883-894.

15. MohlmanJan.A community based survey of older adults' preferences for treatment of anxiety. Psychology \& Aging. 2012; 27:1182.

16. Hunot, V, R Churchill, M Silva de LimaV Teixeira.Psychological therapies for generalised anxiety disorder. Cochrane Database Syst Rev. 2007; 2007:Cd001848.

17. Yovel, I.Acceptance and commitment therapy and the new generation of cognitive behavioral treatments. Isr J Psychiatry Relat Sci. 2009; 46:304-9.

18. Hayes, SC.Acceptance and commitment therapy, relational frame theory, and the third wave of behavioral and cognitive therapies. Behavior Therapy. 2005; 35:639-665.

19. Luoma, JB, SC HayesRD Walser.Learning ACT: An acceptance and commitment therapy skills-training manual for therapists. Psychological Record. 2010; 60:549-552.

20. Hayes, SC, KD Strosahl KG Wilson.Acceptance and commitment therapy: An experiential approach to behavior change. Encyclopedia of Psychotherapy. 1999; 9:1-8.

21. Ruiz, FJ, A Peña-Vargas, ES Ramírez, JC Suárez-Falcón, MB García-Martín, DM García-Beltrán, et al.Efficacy of a two-session repetitive negative thinking-focused acceptance and commitment therapy (ACT) protocol for depression and generalized anxiety disorder: A randomized waitlist control trial. Psychotherapy (Chic). 2020; 57:444-456.

22. Wetherell, JL, N Afari, CR Ayers, JA Stoddard, J Ruberg, JT Sorrell, et al.Acceptance and Commitment Therapy for generalized anxiety disorder in older adults: a preliminary report. Behav Ther. $2011 ; 42: 127-$ 34. 
23. Andrade, H L., Alonso, J., Mneimneh, Z., et al.Barriers to mental health treatment: results from the WHO World Mental Health surveys. PSYCHOLOGICAL MEDICINE -LONDON. 2014.

24. Andersson, GN Titov.Advantages and limitations of Internet-based interventions for common mental disorders. World Psychiatry. 2014; 13:4-11.

25. Wosik, J, M Fudim, B Cameron, ZF Gellad, A Cho, D Phinney, et al.Telehealth transformation: COVID-19 and the rise of virtual care. J Am Med Inform Assoc. 2020; 27:957-962.

26. Bitar, HS Alismail.The role of eHealth, telehealth, and telemedicine for chronic disease patients during COVID-19 pandemic: A rapid systematic review. Digit Health. 2021; 7:20552076211009396.

27. Tse, J, D LaStella, E Chow, E Kingman, S Pearlman, L Valeri, et al.Telehealth Acceptability and Feasibility Among People Served in a Community Behavioral Health System During the COVID-19 Pandemic. Psychiatr Serv. 2021:appips202000623.

28. Hemmings, NR, JM Kawadler, R Whatmough, S Ponzo, A Rossi, D Morelli, et al.Development and Feasibility of a Digital Acceptance and Commitment Therapy-Based Intervention for Generalized Anxiety Disorder: Pilot Acceptability Study. JMIR Form Res. 2021; 5:e21737.

29. Moher, D, L Shamseer, M Clarke, D Ghersi, A Liberati, M Petticrew, et al.Preferred reporting items for systematic review and meta-analysis protocols (PRISMA-P) 2015 statement. Syst Rev. 2015; 4:1.

30. Higgins, JP, DG Altman, PC Gøtzsche, P Jüni, D Moher, AD Oxman, et al.The Cochrane Collaboration's tool for assessing risk of bias in randomised trials. Bmj. 2011; 343:d5928.

31. Guyatt, GH, AD Oxman, GE Vist, R Kunz, Y Falck-Ytter, P Alonso-Coello, et al.GRADE: an emerging consensus on rating quality of evidence and strength of recommendations. Bmj. 2008; 336:924-6.

32. Egger, M, G Davey Smith, M SchneiderC Minder.Bias in meta-analysis detected by a simple, graphical test. Bmj. 1997; 315:629-34.

33. Tyrer, PD Baldwin.Generalised anxiety disorder. Lancet. 2006; 368:2156-66.

34. Dell'Osso, B, G Camuri, B Benatti, M BuoliAC Altamura.Differences in latency to first pharmacological treatment (duration of untreated illness) in anxiety disorders: a study on patients with panic disorder, generalized anxiety disorder and obsessive-compulsive disorder. Early Interv Psychiatry. 2013; 7:374-80.

35. Gersh, E, DJ Hallford, SM Rice, N Kazantzis, H Gersh, B Gersh, et al.Systematic review and metaanalysis of dropout rates in individual psychotherapy for generalized anxiety disorder. J Anxiety Disord. 2017; 52:25-33.

36. Dahlin, M, M Ryberg, K Vernmark, N Annas, P CarlbringG Andersson.Internet-delivered acceptancebased behavior therapy for generalized anxiety disorder: A pilot study. Internet Interv. 2016; 6:16-21. 
37. Kanuri, N, MG Newman, JI Ruzek, E Kuhn, M Manjula, M Jones, et al.The Feasibility, Acceptability, and Efficacy of Delivering Internet-Based Self-Help and Guided Self-Help Interventions for Generalized Anxiety Disorder to Indian University Students: Design of a Randomized Controlled Trial. JMIR Res Protoc. 2015; 4:e136.

38. Dahlin, M, G Andersson, K Magnusson, T Johansson, J Sjögren, A Håkansson, et al.Internet-delivered acceptance-based behaviour therapy for generalized anxiety disorder: A randomized controlled trial.

Behav Res Ther. 2016; 77:86-95.

39. Richards, D, L Timulak, C Rashleigh, O McLoughlin, A Colla, C Joyce, et al.Effectiveness of an internetdelivered intervention for generalized anxiety disorder in routine care: $A$ randomised controlled trial in a student population. Internet Interv. 2016; 6:80-88.

\section{Figures}




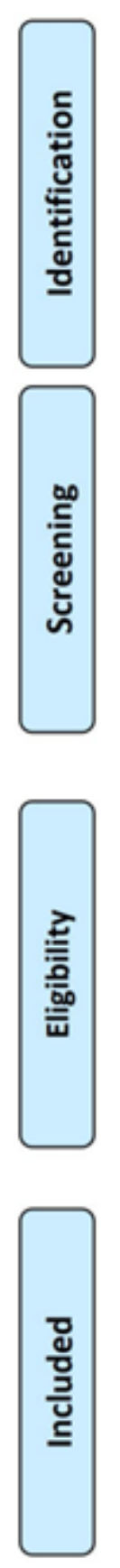

